\title{
Mammalian Genome, volume 5, number 7, 1994, pp 416-423
}

In the article, "Generation and characterization of an ordered lambda clone array for the 460-kb region surrounding the murine Xist sequence," by C. Rougeulle, L. Colleaux, B. Dujon, P. Avner, the legend to Figure 4 (p. 421 ) was inadvertently omitted. Following is the legend to Figure 4:

Fig. 4. Representation of the contig. The fine lines beneath the YAC represent lambda clones and the bold line the fragmented YAC 1Pas9. The physical markers are indicated in italics at the top of the figure and numbers represent the sites of random integration of I-Sce I in the YAC. 